\title{
FEDSM/FMTC-65482
}

\section{PIV VISUALIZATION OF FLOW AROUND AIRFOIL CONTROLLED BY SYNTHETIC JET ACTUATORS AT SHEAR-LAYER AND WAKE INSTABILITY FREQUENCIES}

\author{
Eric Yang \\ Turbulence Research Lab \\ Mechanical and Industrial Engineering \\ University of Toronto \\ Toronto, Ontario 95616 \\ Canada \\ Email: eyang@mie.utoronto.ca
}

\author{
Pierre E. Sullivan \\ Mechanical and Industrial Engineering \\ University of Toronto \\ Toronto, Ontario, M5S 3G8 \\ Canada \\ sullivan@mie.utoronto.ca
}

\begin{abstract}
The response of a separated boundary layer to synthetic jet flow control at the global wake instability $\left(F^{+} \approx \mathscr{O}(1)\right)$ and the shear-layer instability $\left(F^{+} \approx \mathscr{O}(10)\right)$ measured by particle image velocimetry are presented. The visualization shows that in each of the control cases, coherent vorticity develops and breaks down into a turbulent wake. When the jets are actuated by burstmodulation at the wake instability frequency, they induce regular formation and detachment of large-scale vorticity to form a wide turbulent wake. Excitation at the shear-layer instability frequency, on the other hand, produces a train of alternating velocity fluctuations in the boundary layer which dissipate to a narrower wake. Proper orthogonal decomposition of the velocity fields show that the physical extent of the jet-induced coherent structures is decreased with increasing addition of momentum for both excitation frequencies.
\end{abstract}

\section{NOMENCLATURE}

$\alpha \quad$ angle of attack

$b \quad$ span length

$C_{\mathrm{B}}$ blowing ratio

$C_{\mu} \quad$ momentum coefficient

$c \quad$ chord length

$d \quad$ slot width

$F^{+} \quad$ reduced frequency of excitation

$h$ slot span $\theta \quad$ phase angle of actuation cycle

Re chord Reynolds number

$\rho$ density

St Strouhal number

$T \quad$ period of piezoelectric actuation

$u \quad$ horizontal component of velocity

$v \quad$ vertical component of velocity

\section{Introduction}

Flow control using synthetic jet actuators (SJAs) have been shown to be effective at reattaching separated flows over airfoils and effecting lift recovery $[1,2]$. The basic mechanism of control is the formation of a synthetic jet that injects momentum into the shear layer; this enhances mixing and allows the flow to overcome a greater adverse pressure gradient. Research into SJAs is now being pursued towards the development of flow control schemes suitable for real applications (e.g., [3,4]). Despite the progress in this field, effective flow control has not been characterized for the entire parameter space spanned by excitation frequency, blowing ratio, Reynolds number, orifice geometry and angle of attack. The frequency of excitation can be nondimensionalized as the reduced frequency $F^{+}=f_{e} c / U_{\infty}$, which is scaled by the characteristic timescale of the shear layer, while the momentum of the synthetic jet can be quantified using either 
the blowing ratio $C_{\mathrm{B}}=\frac{\bar{u}_{\mathrm{jet}}}{U_{\infty}}$, or the momentum coefficient,

$$
C_{\mu}=\frac{\bar{I}_{\text {jet }}}{\frac{1}{2} \rho_{\infty} U_{\infty}^{2} c}
$$

where

$$
\bar{I}_{\mathrm{jet}}=\frac{2}{T} \rho_{\mathrm{jet}} b \int_{0}^{\frac{T}{2}} u_{\mathrm{jet}}^{2}(t) d t
$$

$d$ is the slot width of the jet orifice, $\rho_{j e t}$ is the fluid density of the jet, $T$ is the period of the piezoelectric actuator motion and $u_{\text {jet }}$ is the average velocity at the jet exit plane. Excitation frequency is one parameter which greatly affects the mechanism of flow reattachment; excitation at the wake instability frequency $\left(F^{+}=\mathscr{O}(1)\right)$ organizes the large-scale vortex shedding, while excitation at the shear-layer instability frequency $\left(F^{+}=\mathscr{O}(10)\right)$ "traps" vorticity in the boundary layer and modifies the aerodynamic shape of the airfoil [5]. Burst-mode modulation has been demonstrated by Amitay et al . to be particularly effective at controlling separated flow [6]. They also found that excitation at the shear-layer instability maximized the ratio of lift to drag, gave to the greatest lift recovery and reattached the flow in a timeaveraged sense [7]. Feero et al. investigated flow control by SJA excitation for $R e=1 \times 10^{5}$ flow around a NACA 0025 airfoil, and showed that the effectiveness of control is improved when the jet is moved towards the leading edge. [8,9]. Just as the authors above, they found that excitation at the shear-layer instability maximized the lift-to-drag ratio; however, it was excitation at the wake instability that gave the greatest lift recovery. This paper aims to illustrate certain aspects of frequency dependency of flow control around a NACA 0025 airfoil. Particle image velocimetry (PIV) was used to measure the velocity field and to visualize the controlled boundary layer and wake. Proper orthogonal decomposition (POD) is applied to the velocity fields to show the coherent structures induced by the SJA control.

\subsection{Methodology}

Measurements were made for flow over a NACA 0025 airfoil installed in a $1.2 \mathrm{~m} \times 0.9 \mathrm{~m}$ recirculating wind tunnel. The airfoil dimensions are shown in Fig. 1, having $c=300 \mathrm{~mm}$ chord-length and $d=885 \mathrm{~mm}$ span. Control was effected by a synthetic jet array composed of 16 piezoelectric disks lining a shared cavity underneath the airfoil surface at $x / c=0.12$, with a single slot for an orifice with dimensions $d=0.5 \mathrm{~mm}, d=294$ $\mathrm{mm}$ spanwise extent and $5 \mathrm{~mm}$ depth. The freestream velocity was $R e_{c}=100,000\left(U_{\infty} \approx 5 \mathrm{~m} / \mathrm{s}\right)$ with a turbulence intensity of $0.08 \%$. The SJAs were driven by a $1000 \mathrm{~Hz}$ sine wave applied to the piezoelectric elements, giving a jet excitation frequency of

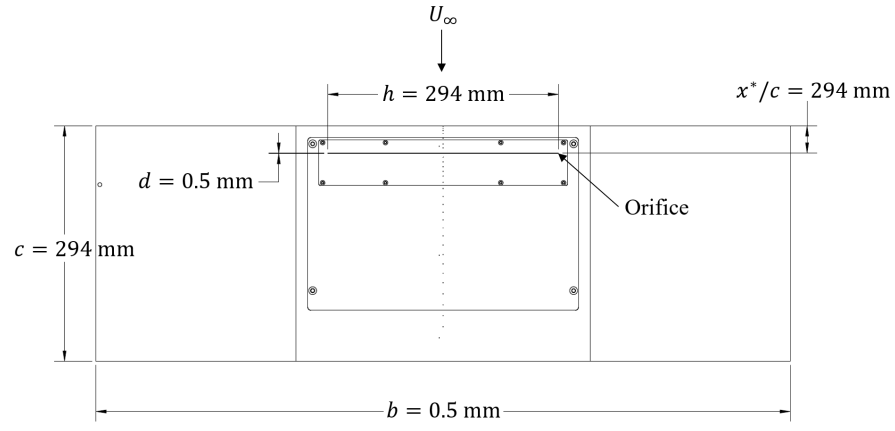

FIGURE 1: LAYOUT OF SYNTHETIC JET SLOT ON NACA 0025 AIRFOIL.

$F^{+}=58$ that is burst-modulated to lower values of values $F^{+}$ using square waves. Phase-averaged velocity fields were measured by PIV for 8 points (spaced $45^{\circ}$ apart) in the modulation cycle, for $F^{+}=1$ and $F^{+}=14$. PIV images were captured for a section of the flow over the top surface of the airfoil by two 5 MP, sCMOS cameras with $60 \mathrm{~mm}$ lenses set to $2.8 \mathrm{f}$-stop, giving a resolution of 11.7 pixels per $\mathrm{mm}$. The flow was seeded with DEHS (sebecate) particles generated by an aerosol generator and the measurement plane at midspan was illuminated by a dual-cavity $200 \mathrm{~mJ}, 532 \mathrm{~nm}$ Evergreen laser with a $90 \mu$ s time separation. During the experiments, 500 image pairs were captured by each camera for each control case and phase angle. The PIV images were processed by a GPU-based, iterative correlation algorithm developed in part by the authors and the OpenPIV project $[10,11]$. A resolution of $0.34 \mathrm{~mm}$ between vectors was attained using $8 \times 8$ correlation windows. The field of view for the two cameras were overlapped by $30 \%$ and the resulting velocity fields from each camera were composited by weighting the vectors by a modified sigmoid in the overlapping region (composited area is shown in Fig. 3).

\section{Results and Discussions \\ 2.1 Flow visualization}

For uncontrolled flow over the airfoil at $R e=1 \times 10^{5}$ and $\alpha=12^{\circ}$, the flow was separated just past the suction peak at the top of the airfoil. It was previously found that for this flow configuration that $C_{\mathrm{B}}=0.1$ was the threshold momentum required to reattach the flow in a time-averaged sense [8]. When excitation is applied at and beyond the threshold $C_{\mathrm{B}}$, the flow over the airfoil was reattached for all cases measured. As was stated in the introduction, two types of flow reattachment are expected when synthetic jet excitation is applied: organization of large-scale vortex shedding from exciting the global wake instability and the creation of smaller-scale vorticity in the boundary layer from exciting the shear-layer instability. Figure 2 shows the streamlines 


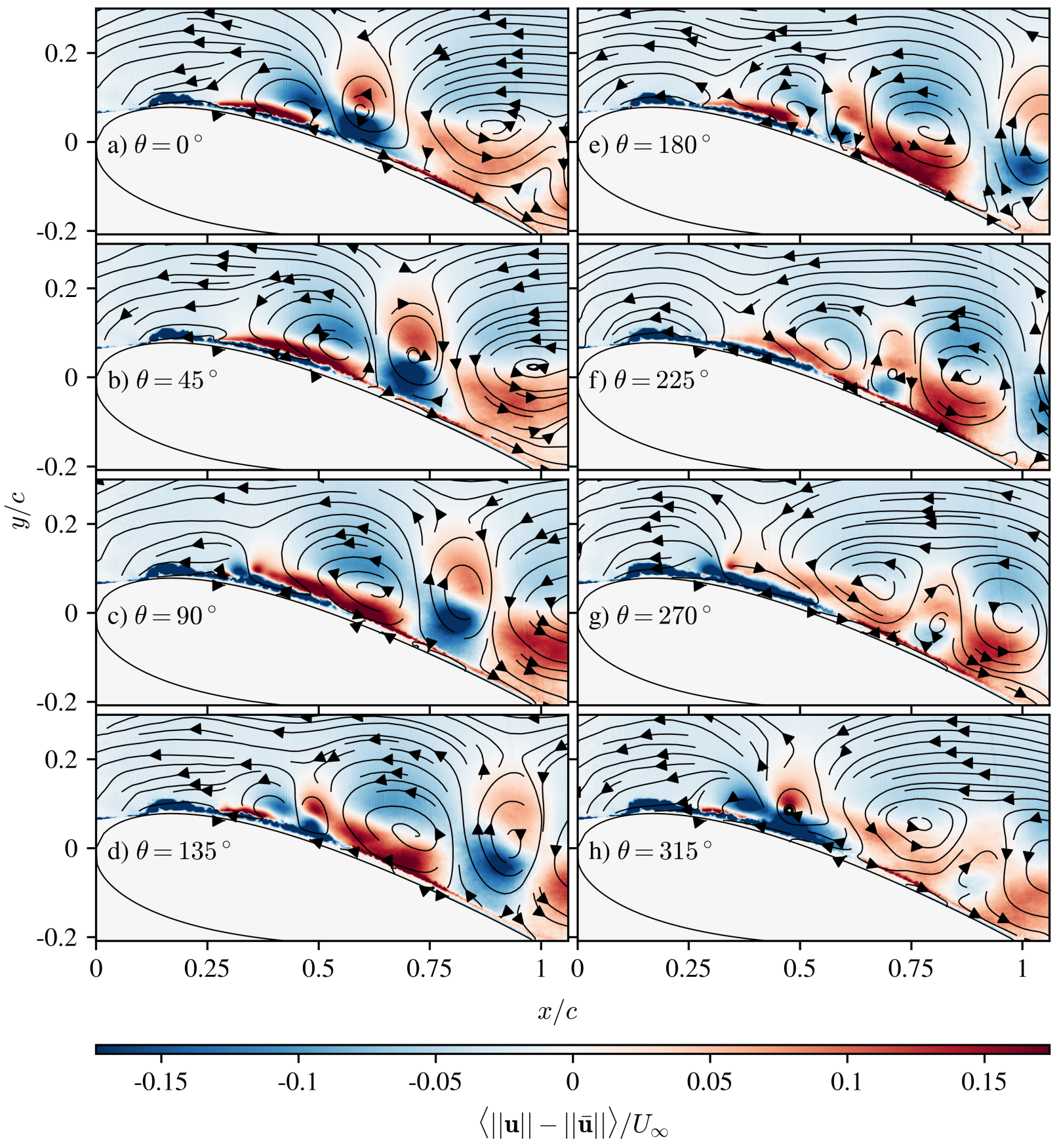

FIGURE 2: PHASE-AVERAGED STREAMLINES FOR $F^{+}=1$ WITH TIME-AVERAGED FLOW SUBTRACTED..

of flow controlled by excitation at the wake instability frequency $F^{+}=1$ and $C_{\mathrm{B}}=0.1$ over the airfoil for every phase angle measured. The time-averaged averaged flow has been subtracted (triple-decomposed) to visualize the circulation of the eddies as they detach at a point just past the suction peak and advect into the wake. As seen from the phase-averaged streamlines, the control results in the organized shedding of vorticity, similar to the results of the literature $[6,12]$. One primary vortex advects away each actuation cycle at $S t=F^{+}$, which is followed by smaller eddies in a counter-rotating train. At about $\theta=270^{\circ}, 2 \mathrm{~g}$ ), the primary counter-clockwise vortex begins to form, visualized as a structure with a regions of positive triple-decomposed velocity above a negative-signed one. Lesser-intensity counter-rotating appear following the first ones with decreasing strength, with the second counter-clockwise one appearing by $\theta=135^{\circ}$. When this flow is time-averaged, however, the flow is reattached and 


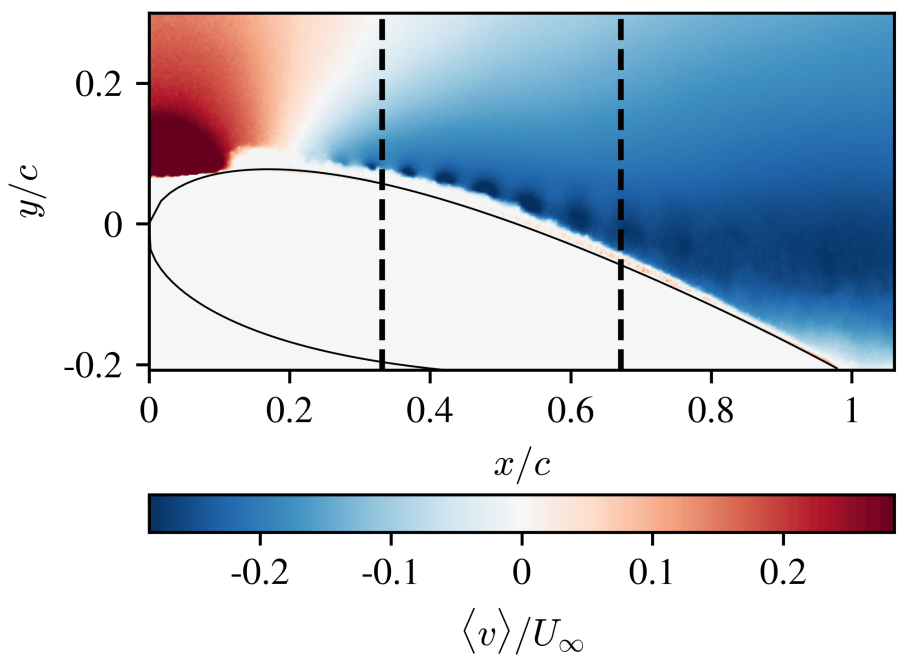

FIGURE 3: $v$-COMPONENT OF VELOCITY FIELD FOR $F^{+}=14-$ CONTROL AT $\theta=0^{\circ}$. DASHED LINES BOUND THE COMPOSITED MEASUREMENT DOMAIN.

there is a large turbulent wake that can seen by the plot of turbulent kinetic energy shown in Fig. 4a. It is suggested that the large-scale mixing caused by the passage of eddies in the wake aids the flow reattachment by entraining fluid into the boundary layer [13]. The vortices grow in spatial extent as they advect downstream, which increases the height of the wake progressing down the chord. For excitation at the shear layer instability $F^{+}=14$ and $C_{\mathrm{B}}=0.1$, the response is virtually time-invariant with respect to the time scales of the shear layer. Induced vortical structure in the shear layer by the control are visualized by periodic disturbances in the phase-averaged $v$-field, shown in Fig. 3. The existence of the vortices is confirmed by positive regions in the Q-criterion-field [14], which indicates that the vortices persist to $x / c \approx 0.6$, where they dissipate into general shear-layer vorticity. For the lifetime of the vortices, they remain in the boundary layer and follow the airfoil profile. It is notable that the point $x / c \approx 0.6$ where the vortices dissipate in the boundary layer roughly corresponds to where a turbulent mixing layer diverges from the airfoil profile at $x / c \approx 0.55$ and at an angle of $\approx 10^{\circ}$. The wake of the airfoil under this control is shown by the TKE field in $4 \mathrm{a}$, where the turbulent wake develops at $x / c \approx 0.55$ and the overall size of the wake is significantly smaller than for control at $F^{+}=1$.

\subsection{Coherent structures from POD}

Proper orthogonal decomposition was performed on the time-averaged data for varying $F^{+}$and $C_{\mathrm{B}}$, which decomposes the fluctuations of the flow into its most energy-containing modes. The distribution of turbulent kinetic energy in the POD modes are shown in Fig. 5 for $C_{\mathrm{B}}=0.1$ and $C_{\mathrm{B}}=1$. The POD energy distributions exhibit a trend where excitation at higher $F^{+}$

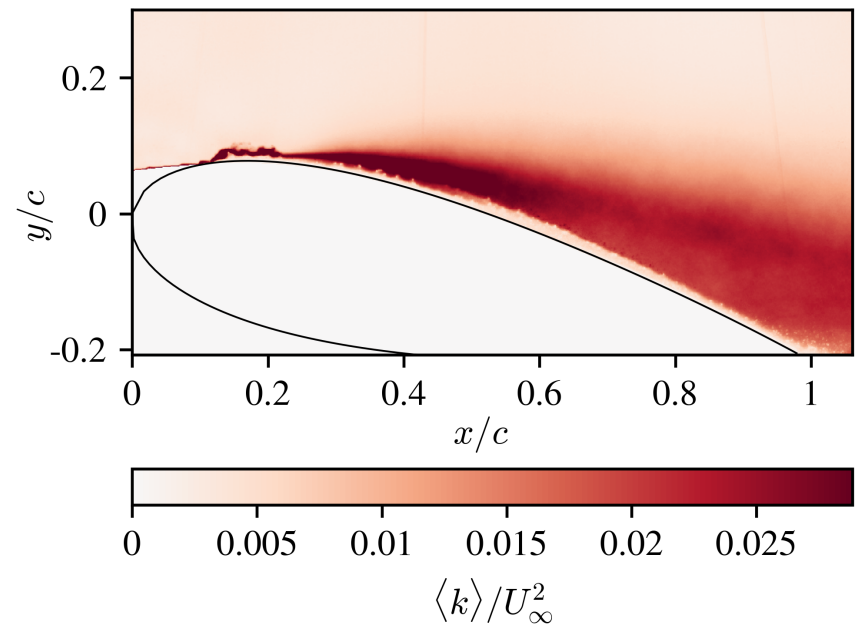

(a) $F^{+}=1$

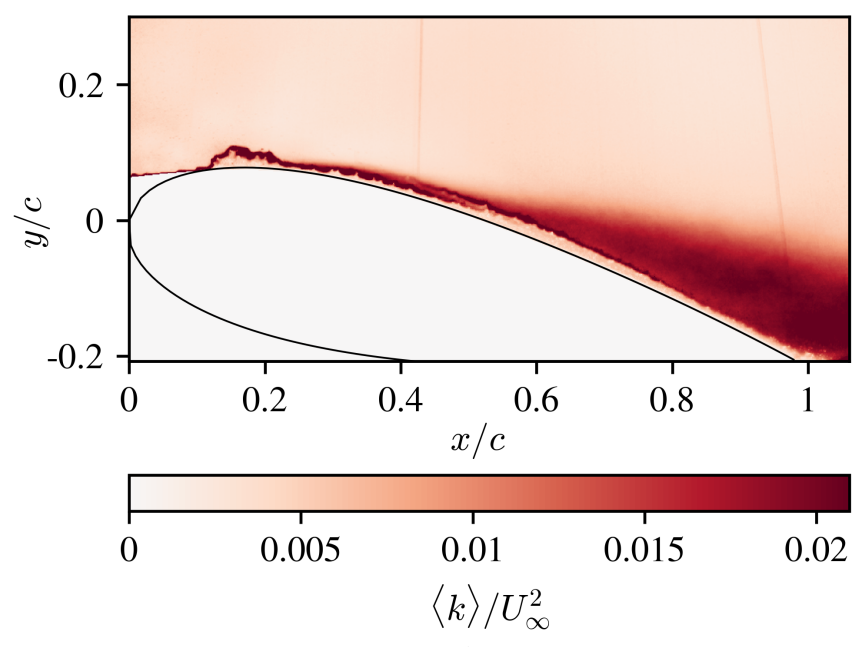

(b) $F^{+}=14$

FIGURE 4: TIME-AVERAGED TURBULENT KINETIC ENERGY.

reduces the turbulent energy contained in the first POD modes for both $C_{\mathrm{B}}=0.1$ and $C_{\mathrm{B}}=1$. From this and the above discussion on control-induced vortices, it can be inferred that the proportion energy contained in the most energetic modes is related to the physical scale of the vortical structures in the flow. For the $F^{+}=1$ wake-instability excitation, the large eddies detaching into the wake result in highly-energetic first modes, while for $F^{+}=14$, the train of vortices close to the airfoil surface contain much less coherence in the first modes. Increasing the momentum of the excitation also decreases the coherence of the first modes. It is notable that excitation at $F^{+}=58$ does not result in even less coherence than for the lower case of $C_{\mathrm{B}}=1$, $F^{+}=14$. The difference may be attributable to the lack of burstmodulation for $F^{+}=58$-control, which has different behaviour than burst-modulated control at the same $F^{+}[6]$. In the baseline flow, there the coherence results from shedding of medium-scale 


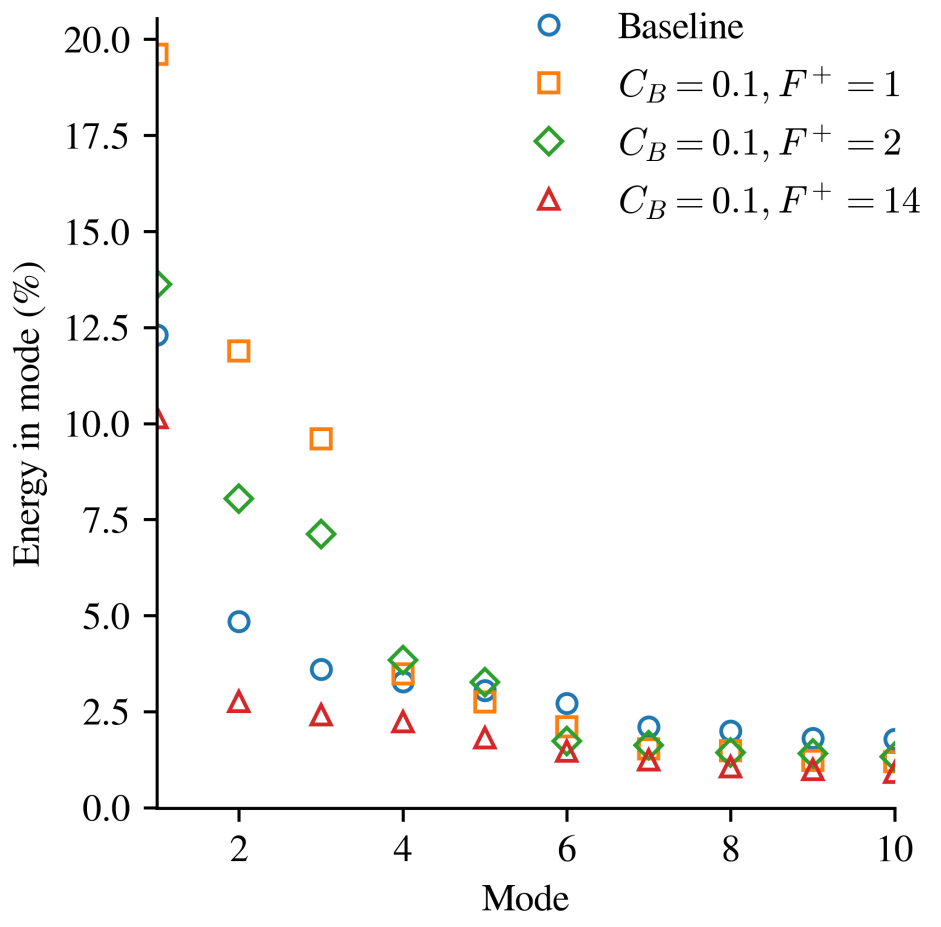

(a) $C_{\mathrm{B}}=0.1$

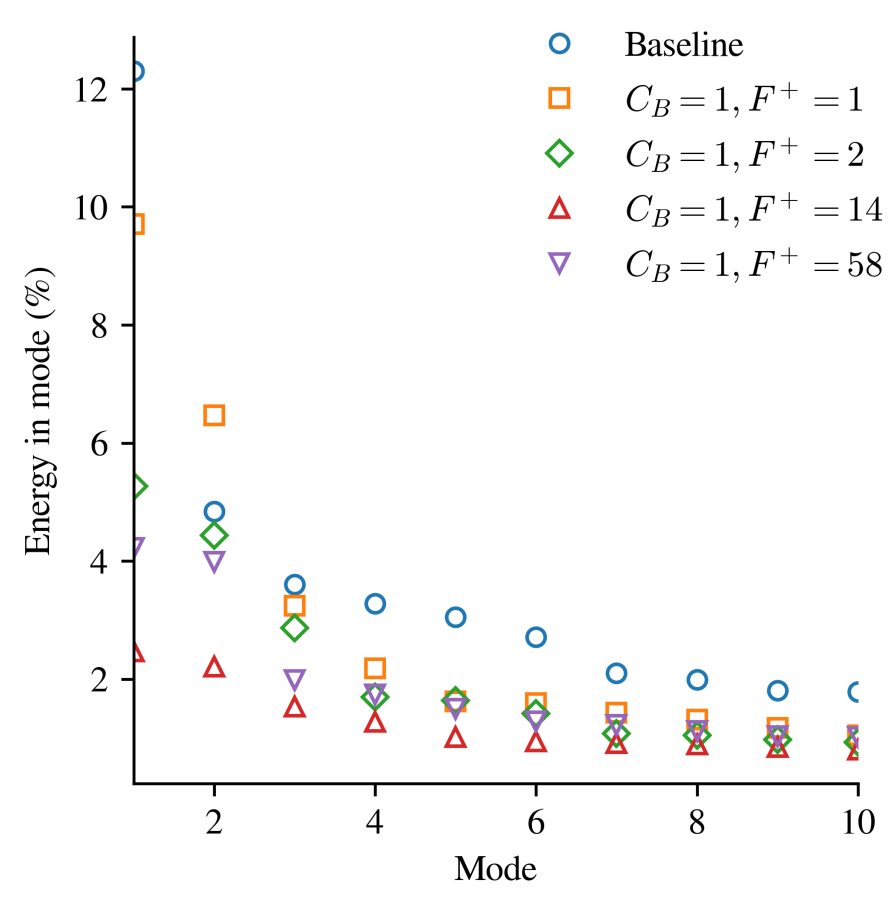

(b) $C_{\mathrm{B}}=1$

FIGURE 5: POD ENERGY CONTAINED IN FIRST 10 MODES.

eddies at the detachment point. The first POD mode is shown for illustrative purposes in Fig. 6 for control at $F^{+}=14$ and both $C_{\mathrm{B}}=0.1$ and $C_{\mathrm{B}}=0.8$. It is seen that control at the shear layer instability introduces a train of coherent structures of alternating sign past the synthetic jet actuation location. The coherent structures appear near the synthetic jet orifice and begin to break down midway down the chord. As $C_{\mathrm{B}}$ is increased, the scale of the correlated regions in the first modes decreases, in agreement with the energy distributions above, so that by $C_{\mathrm{B}}=1$ it becomes difficult to distinguish the coherence in the boundary layer.

\section{Conclusions}

The effects of flow control on the shear layer and wake of $R e=1 \times 10^{5}$ flow around a NACA 0025 airfoil have been visualized by phase- and time-averaged PIV at measurements at the midspan. Control at the wake instability frequency organizes the shedding of large vortices that create a large wake in the turbulent field; the vortices induced by control at the shear-layer instability remain in the shear-layer and dissipate to form a much narrower wake. The results support the idea of excitation frequency as the parameter determining the time-dependency of the shear-layer response, as well the the size of the wake generated by vorticity. POD has also been used to visualize the scale of coherence induced by SJA flow control. Coherent structures in the boundary layer are reduced in size with increasing $F^{+}$and $C_{\mathrm{B}}$. While $F^{+}$affects the evolution of the vortices induced by con- trol, the role of $C_{\mathrm{B}}$ on the size of coherent structures should also be a research interest. From previous studies, it was established that excitation at the shear layer instability $f=\mathscr{O}(10)$ effects a time-invariant reattachment of the flow and maximizes lift-todrag. However whether wake-instability excitation or shear-layer excitation maximizes the absolute recovery to lift remains to be determined for all flow configurations. Research incorporating these control parameters should be undertaken to develop a robust control scheme for real flight-applications.

\section{ACKNOWLEDGMENT}

The authors would like to acknowledge the support of the Natural Sciences and Engineering Research Council of Canada and the University of Toronto Faculty of Applied Science and Engineering. This research was enabled in part by support provided by Compute Ontario and Compute Canada. Computations were performed on the MIST supercomputer at the SciNet HPC Consortium. SciNet is funded by: the Canada Foundation for Innovation; the Government of Ontario; Ontario Research Fund Research Excellence; and the University of Toronto.

\section{REFERENCES}

[1] Greenblatt, D., and Wygnanski, I. J., 2000. "Control of flow separation by periodic excitation".

[2] Chovet, C., Lippert, M., Foucaut, J.-M., and Keirsbulck, L., 


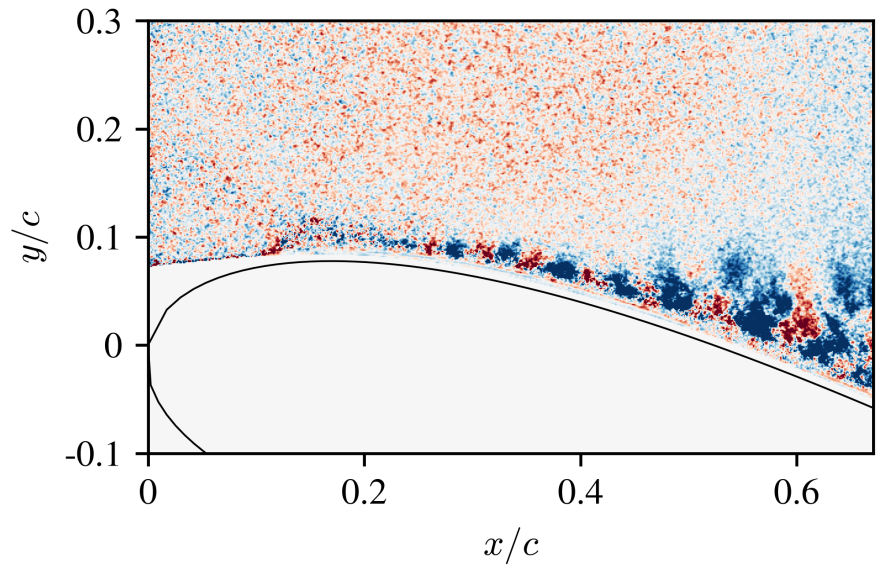

(a) $C_{\mathrm{B}}=0.1, F^{+}=14$.

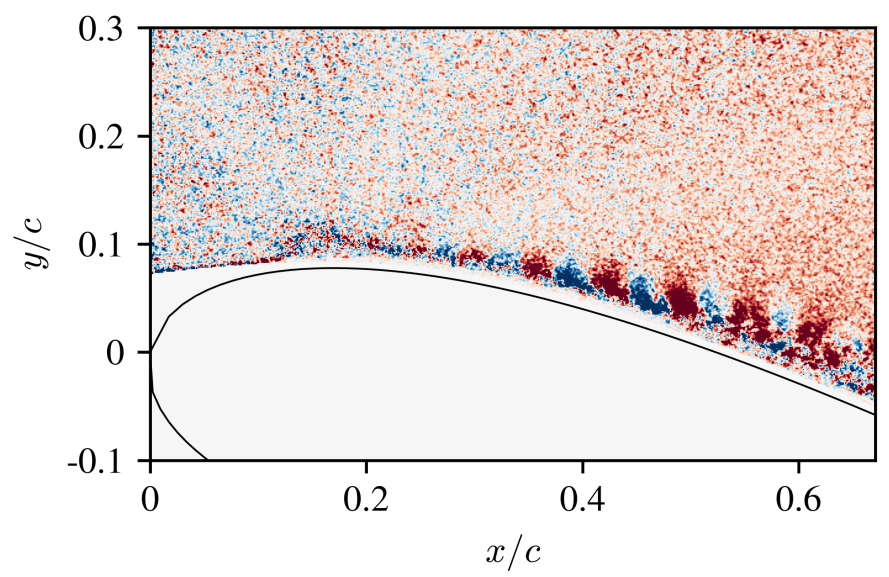

(b) $C_{\mathrm{B}}=0.8, F^{+}=14$.

FIGURE 6: FIRST POD MODE FOR $v$-COMPONENT OF FLOW OVER AIRFOIL. COLORS INDICATE THE DEGREE OF CORRELATION.

2017. "Dynamical aspects of a backward-facing step flow at large reynolds numbers". Experiments in Fluids, 58(11), pp. $1-15$.

[3] Deem, E. A., Cattafesta, L. N., Hemati, M. S., Zhang, H., Rowley, C., and Mittal, R., 2020. "Adaptive separation control of a laminar boundary layer using online dynamic mode decomposition". Journal of Fluid Mechanics.

[4] Pinier, J. T., Ausseur, J. M., Glauser, M. N., and Higuchi, H., 2007. "Proportional closed-loop feedback control of flow separation". AIAA Journal.

[5] Glezer, A., 2011. Some aspects of aerodynamic flow control using synthetic-jet actuation.

[6] Amitay, M., and Glezer, A., 2002. “Controlled transients of flow reattachment over stalled airfoils". International Journal of Heat and Fluid Flow.

[7] Amitay, M., Smith, D. R., Kibens, V., Parekh, D. E., and
Glezer, A., 2001. "Aerodynamic flow control over an unconventional airfoil using synthetic jet actuators". AIAA journal.

[8] Feero, M. A., Goodfellow, S. D., Lavoie, P., and Sullivan, P. E., 2015. "Flow reattachment using synthetic jet actuation on a low-reynolds-number airfoil". AIAA Journal.

[9] Feero, M. A., Lavoie, P., and Sullivan, P. E., 2017. "Threedimensional span effects of high-aspect ratio synthetic jet forcing for separation control on a low Reynolds number airfoil". Journal of Visualization, 20(1), feb, pp. 45-51.

[10] Liberzon, A., Gurka, R., Taylor, Z. J., Lasagna, D., Aubert, M., Bachant, P., Dallas, C., Käufer, T., Borg, J., Dallas, C., Curry, C., and Vodenicharski, B., 2019. OpenPIV.

[11] Dallas, C., Wu, M., Chou, V., Liberzon, A., and Sullivan, P. E., 2019. "Graphical Processing Unit-Accelerated OpenSource Particle Image Velocimetry Software for High Performance Computing Systems". Journal of Fluids Engineering, Transactions of the ASME.

[12] Smith, B. L., and Glezer, A., 1998. "The formation and evolution of synthetic jets". Physics of Fluids, 10(9), sep, p. 2281.

[13] Buchmann, N. A., Atkinson, C., and Soria, J., 2013. "Influence of ZNMF jet flow control on the spatio-temporal flow structure over a NACA-0015 airfoil". Experiments in Fluids.

[14] Dubief, Y., and Delcayre, F., 2000. "On coherent-vortex identification in turbulence". Journal of turbulence, $\mathbf{1}$, pp. 011-011. 